\title{
Cardiothoracic surgery training in Australia and New Zealand
}

William Y. Shi, MBBS, PhD, ${ }^{\text {a,b }}$ Zaita Oldfield, MEd, ${ }^{\mathrm{c}}$ Robert Tam, MBBS, FRACS, ${ }^{\mathrm{c}}$ Andrew D. Cochrane, MBBS, FRACS, ${ }^{d}$ and Julian A. Smith, MBBS, MS, FRACS, FACS ${ }^{\mathrm{d}}$

From the ${ }^{\mathrm{a}}$ The Alfred Hospital, Melbourne, Australia; ${ }^{\mathrm{b}}$ Melbourne Medical School, Faculty of Medicine, University of Melbourne, Melbourne, Australia; ${ }^{c}$ Royal Australasian College of Surgeons, East Melbourne, Australia; and ${ }^{\mathrm{d} D e p a r t m e n t}$ of Surgery (School of Clinical Sciences at Monash Health), Monash University, Melbourne, Australia.

Received for publication Jan 26, 2018; revisions received March 13, 2018; accepted for publication March 16, 2018; available ahead of print May 3, 2018.

Address for reprints: Julian A. Smith, MBBS, MS, FRACS, FACS, Department of Surgery (School of Clinical Sciences), Level 5, Block E Monash Medical Centre, 246 Clayton Rd, Clayton, Victoria 3168, Australia (E-mail: julian.smith@monash.edu).

J Thorac Cardiovasc Surg 2018;156:718-25

$0022-5223 / \$ 36.00$

Copyright (c) 2018 by The American Association for Thoracic Surgery https://doi.org/10.1016/j.jtcvs.2018.03.086

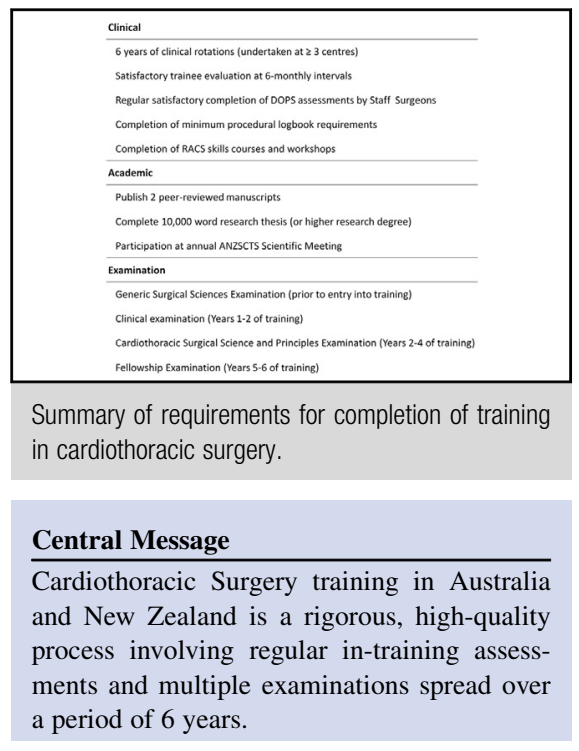

See Editorial Commentary page 726

See Editorial page 716. Sydney.

Several surgeons from Australia and New Zealand have made important contributions within the cardiac surgical domain. Sir Brian Barratt-Boyes of the famed Greenlane Hospital in Auckland was a pioneer in adult and pediatric cardiac surgery, especially with the use of aortic homograft replacement. Dr Roger Mee brought the cardiac surgery program at Royal Children's Hospital in Melbourne to international attention with its outstanding clinical results. Dr Brian Buxton and Dr James Tatoulis have spearheaded and published widely on the use of arterial conduits in coronary surgery, for which the city of Melbourne is now well known on the world stage. Dr Mark O'Brien of Brisbane was a leading exponent of cryopreserved allograft replacement of the aortic valve and root. Dr Victor Chang established heart and lung transplantation in Australia at St Vincent's Hospital in Sydney. His colleague Dr Donald Esmore subsequently established heart and lung transplantation at the Alfred Hospital in Melbourne, which now houses one the world's largest and most successful lung transplant programs.

Australia has a population of approximately 25 million people, with $27 \%$ of its population aged more than 55 years. Australians have an average life expectancy at birth of 81 years. New Zealand has a population of 4.8 million. Like Australia, 27\% of its population is aged more than 55 years, and the average life expectancy is also 81 years. ${ }^{2,3}$

The Australia and New Zealand Society of Cardiac and Thoracic Surgeons (ANZSCTS) Database ${ }^{4}$ was established in 2001 and collects data on 39 public $(n=23)$ and private $(\mathrm{n}=16)$ cardiac surgery centers across Australia. There are 19 cardiac surgery centers that are not yet participating in the database. A lack of logistic and infrastructure support at smaller cardiac surgery centers has limited their participation in the database. New Zealand centers are also yet to join the database. According to the database, approximately 12,000 cardiac surgeries are performed per year, and 107,249 cardiac surgeries are currently recorded in the database. The caseload and case mix per unit and per year are presented in Figures 1 and 2. ${ }^{5}$ At present, there is no centralized database that collects information on thoracic surgical procedures, although this is currently in development.

There are currently approximately 180 cardiothoracic surgeons in Australia and New Zealand. Although there are no formal data on individuals' spectrum of practice across Australia and New Zealand, we estimate that 


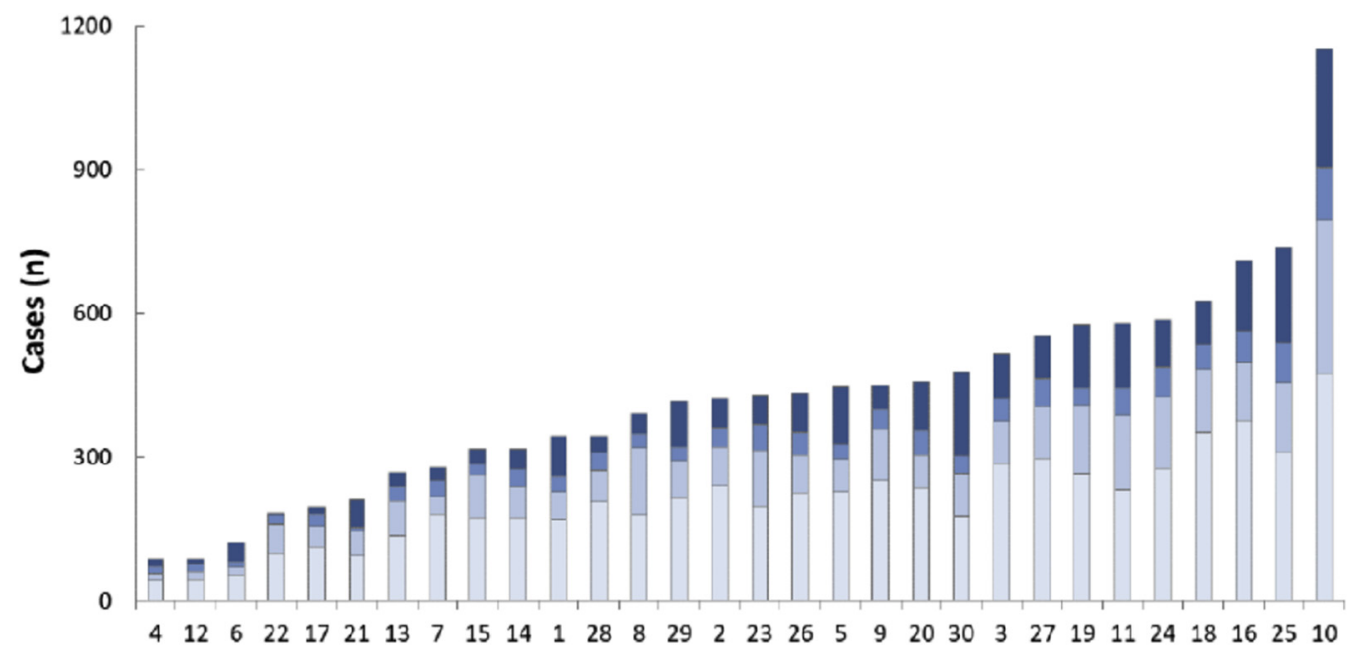

FIGURE 1. Cardiac procedures performed by unit. Reproduced with permissions, ANZSCTS Database Steering Committee, Annual Report 2016. ${ }^{5}$

approximately $75 \%$ of surgeons practice predominantly adult cardiac surgery, whereas $25 \%$ focus their practice mainly on general thoracic surgery. Between our 2 countries, there are 15 pediatric congenital cardiac surgeons, concentrated at 5 major centers.

\section{ROYAL AUSTRALASIAN COLLEGE OF SURGEONS}

The Royal Australasian College of Surgeons (RACS) was founded in 1927 and for more than 80 years has served as the authoritative body for standards and training in surgery in Australia and New Zealand. The RACS's mission is to train surgeons to become competent, independently practicing specialists in each of the 9 recognized specialties: Cardiothoracic Surgery, General Surgery, Neurosurgery, Orthopaedic Surgery, Otolaryngology, Head and Neck Surgery, Pediatric Surgery, Plastic and Reconstructive Surgery, and Urology and Vascular Surgery. The RACS is accredited by the Australian Medical Council and the Medical Council of New Zealand to deliver specialist surgical education, training, and professional development programs.

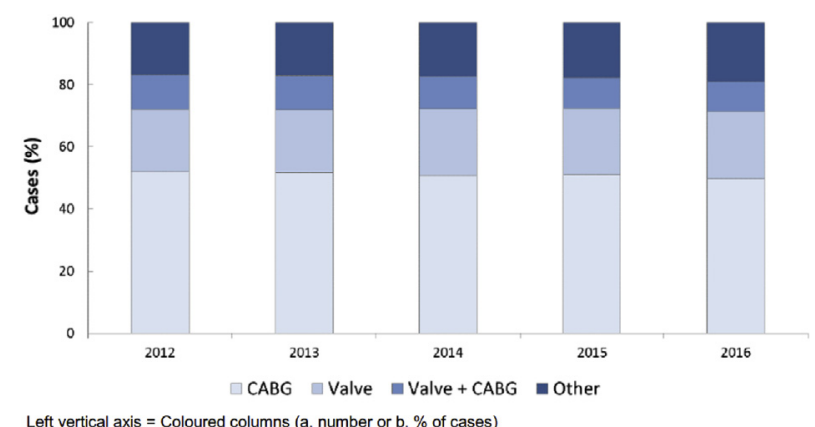

FIGURE 2. Cardiac procedures performed by year. $C A B G$, Coronary artery bypass grafting. Reproduced with permissions, ANZSCTS Database Steering Committee, Annual Report 2016. ${ }^{5}$
The RACS Surgical Education and Training (SET) program provides education and formal training for trainees. The SET program is designed to allow early selection into specialty training, whereby successful applicants are selected directly into a specialty 2 to 4 years after graduating from medical school. Common RACS competencies and standards underpin training across all specialties. In the past, trainees typically undertook a period of generic basic surgical training before their entrance into subspecialty training. However, the implementation of the SET program 10 years ago ensures direct entry into one of the 9 specialties.

\section{CARDIOTHORACIC SURGERY TRAINING PROGRAM OVERVIEW}

The RACS SET program in Cardiothoracic Surgery provides trainees with clinical and operative experience in both cardiac and thoracic surgical conditions. The training program is generally undertaken sequentially over a 6-year period. Upon graduating from medical school, individuals generally undertake 3 to 4 years of hospital rotations before commencing the formal 6-year training program. As such, it generally takes a total of 9 to 10 years to complete Cardiothoracic Surgery training after medical school. After this formal training period, graduates of the program may thereafter pursue additional fellowship experience locally or abroad in areas such as congenital surgery, heart failure/ transplantation, minimally invasive surgery, thoracic aortic surgery, surgical oncology, and so forth.

Trainees' clinical experiences are supplemented by regular courses run by the RACS and ANZSCTS. In most departments, trainees receive weekly education sessions and participate in outcomes audit and mortality/morbidity conferences.

The training program produces independent practitioners of both Adult Cardiac Surgery and General Thoracic 
Surgery. Although surgeons may subsequently concentrate their practice upon cardiac or thoracic surgery, the training program combines both domains. In Australia and New Zealand, esophageal and foregut surgery is generally performed by Upper Gastrointestinal Surgeons; as such, the training in these fields is relatively limited for cardiothoracic trainees. Exposure to pediatric and adult congenital cardiac surgery is not a mandatory component of the training program, and those wanting to pursue this area generally undertake additional post-Fellowship subspecialty training. At the satisfactory conclusion of training, Fellowship of the Royal Australasian College of Surgeons (FRACS) in Cardiothoracic Surgery is expected to be awarded. Training is overseen by the Board of Cardiothoracic Surgery, which consists of 13 members, including 1 trainee representative. The Board is led by its Chairperson.

\section{PREREQUISITE TRAINING}

In Australia and New Zealand, medical school graduates must complete an internship year as their first postgraduate year (PGY1). Once the internship has been completed and General Medical Registration has been obtained, junior doctors progress to PGY2, which is the first year in which doctors are eligible to apply to the RACS SET program. Successful applicants would thereafter commence their surgical training in PGY3. Each surgical specialty has specific prerequisites, and the ability to successfully gain entry into the SET program depends more on meeting these requirements than merely relying on the PGY level. For the SET program in Cardiothoracic Surgery, it is unusual to commence training in PGY3, and trainees typically commence the formal 6-year program in PGY4 or beyond.

The years between internship and the commencement of training are generally spent accruing experience and gaining additional clinical responsibilities as junior resident medical officers and junior "unaccredited" registrars in various specialty rotations. During this time, many individuals undertake research projects or a higher research degree (Masters or $\mathrm{PhD}$ ) to both foster their interest and to mount a competitive application for surgical training. Formal training in General Surgery is not a prerequisite for entry into Cardiothoracic Surgery.

\section{SELECTION INTO CARDIOTHORACIC SURGERY TRAINING}

The requirements for selection into training are summarized in Table 1. The RACS SET program in Cardiothoracic Surgery typically accepts 5 to 8 of 40 to 50 applicants across Australia and New Zealand each year. In 2017, there were 45 applicants of whom 8 were selected to enter the training program. Passing the RACS Generic Surgical Sciences Examination (GSSE) is a requirement before application to the SET program. The GSSE examines knowledge in anatomy,
TABLE 1. Requirements for selection into the Royal Australasian College of Surgeons training program in Cardiothoracic Surgery

Basic requirements

Permanent residency or citizenship of Australia or New Zealand

General (unconditional) medical registration

Pass in the generic surgical sciences examination

Minimum clinical requirements

2 surgical terms of $\geq 10 \mathrm{wk}$ in duration

1 cardiothoracic term $\geq 10 \mathrm{wk}$ in duration

Complete DOPS assessment for:

- Chest drain insertion

- Saphenous vein harvest

- Radial artery harvest

Selection criteria

Structured curriculum vitae $(20 \%)$

Structured referee reports $(35 \%)$

Semistructured interview $(45 \%)$

DOPS, Direct Observation of Procedural Skills.

pathology, and physiology, and is a common examination taken by prospective trainees of all RACS specialties.

Applicants must also have completed a rotating term as a junior resident medical officer in Cardiothoracic Surgery and provide documentation of their ability to performindependently or under supervision-saphenous vein harvest, radial artery harvest, and chest drain insertion. Applicants are scored on their curriculum vitae (20\%), structured referee reports $(35 \%)$, and semistructured interview performance $(45 \%)$. Active trainees in one RACS surgical specialty are permitted to apply mid-training to another specialty as part of the annual selection process, and, if successful, transfer training programs.

All RACS SET training programs are open to International Medical Graduates (IMGs) provided the application prerequisites are met. The RACS has a separate system for awarding Fellowship to those who have already completed specialty surgical training in another country. IMGs who do not undertake the standard SET program may be admitted to Fellowship via examination or assessment depending on their prior experience and level of competence. This process is presented in greater detail on the RACS website (www. surgeons.org). Individuals seeking to undertake postgraduate fellowships in areas such as congenital heart surgery and transplantation, among others, do not need to be a permanent resident. However, these fellowships exist outside the formal training program administered by the RACS. In 2017, the Board approved the RACS policy to increase the recruitment and selection of Aboriginal, Torres Strait Islander, or Maori trainees into Cardiothoracic training.

\section{CARDIOTHORACIC SURGERY TRAINING POSITIONS}

Trainees are allocated to positions within various hospital systems in Australia or New Zealand by the RACS Board of 
TABLE 2. Criteria for minimum operative experience prescribed by the Royal Australasian College of Surgeons Board of Cardiothoracic Surgery

\begin{tabular}{lc}
\hline \multicolumn{1}{c}{ Procedure/component } & N \\
\hline Aortocoronary anastomosis & 75 \\
Cannulation for bypass & 50 \\
Distal coronary anastomosis & 75 \\
Insertion of coronary sinus & 50 \\
$\quad$ cannula &
\end{tabular}

Internal thoracic artery

harvest

Median sternotomy

Radial artery harvest

Redo sternotomy

Saphenous vein harvest

Sternal closure

200

Coronary artery bypass

Unassisted*

Trainee assisted*

First assistant*

Aortic valve surgery

Trainee assisted

First assistant

Aortic surgery

First assistant

Mitral valve surgery

Trainee assistant

First assistant

Other valve surgery

First assistant

Pacemakers

Trainee assisted

Total major cardiac procedures

Trainee unassisted

Trainee assisted

First assistant

Thoracotomy \pm lung biopsy

Trainee unassisted

Trainee assisted

First assistant

Pulmonary resection

Trainee unassisted

Trainee assisted

First assistant

Total major thoracic procedures

Trainee unassisted

Trainee assisted

First assistant
TABLE 2. Continued

\begin{tabular}{lc}
\hline Procedure/component & $\mathbf{N}$ \\
\hline VATS procedures & \\
Trainee unassisted & 20 \\
Trainee assisted & 50 \\
Bronchoscopy & \\
Trainee assisted/unassisted & 80 \\
\hline
\end{tabular}

VATS, Video-assisted thoracic surgery. *Trainee unassisted: Trainee performs the case as primary surgeon with the consultant surgeon unscrubbed. The trainee's assistant is of equal seniority or junior to the trainee; Trainee assisted: Trainee performs the case as primary surgeon, assisted by the consultant surgeon, or a more senior trainee; First assistant: The trainee is the first assistant for the case (on the left side of the operating table assisting the consultant, or a more senior colleague.

Cardiothoracic Surgery for periods of time ranging between 6 months and 2 years. Trainees may spend a maximum of 2 years at any given hospital. In general, trainees spend 1 to 2 years at centers that perform both cardiac and thoracic surgery, whereas 6- to 12-month terms are reserved for pure thoracic surgery or pediatric cardiac surgery rotations. Over the 6-year training period, trainees generally spend time at 3 or 4 centers, which may be located in cities across Australia and New Zealand. This model is designed to give trainees a wide range of clinical and operative experiences. Trainees may select terms at specific hospitals to gain greater exposure in their area of interest as long as core requirements of training in adult cardiac and general thoracic surgery are fulfilled.

There are currently 38 training positions across 30 centers (25 in Australia and 5 in New Zealand). Of these, 3 are for pure thoracic surgery and 2 are for pediatric cardiac surgery. Four posts are pure cardiac rotations. The remaining positions combine elements of adult cardiac and general thoracic surgery.

Over the years, some posts in pure thoracic and pediatric cardiac surgery have been unfilled because of requirements and preferences within the trainee cohort (ie, a trainee may want to gain additional exposure and experience in adult cardiac surgery and be reluctant to lose momentum in their training by undertaking thoracic or pediatric terms). Such posts may be staffed by local or international postgraduate fellows seeking to gain further experience in these fields. At the time of writing, 1 pediatric cardiac surgery post is unfilled, whereas all remaining posts are occupied.

There are usually 35 to 40 active trainees across Australia and New Zealand at various levels of training. There are approximately 5 or fewer IMGs under supervision at any one time, although they are not formally classified as trainees.

Each training unit is accredited by the Board to provide training. The accreditation process is by way of hospital inspection, interviews with stake holders, and assessment of 
TABLE 3. Guidelines for minimum operative experience by the end of the third year of training (SET 3)

\begin{tabular}{lr}
\hline \multicolumn{1}{c}{ Procedure/component } & N \\
\hline Aortocoronary anastomosis & 10 \\
Cannulation for bypass & 50 \\
\hline Internal thoracic artery harvest & 50 \\
\hline Median sternotomy & 80 \\
\hline Radial artery harvest & 10 \\
Saphenous vein harvest & 125 \\
\hline Sternal closure & 80 \\
\hline Coronary artery bypass & 150 \\
$\quad$ First assistant & \\
\hline Aortic valve surgery & \\
$\quad$ First assistant & 20 \\
\hline Mitral valve surgery & \\
$\quad$ First assistant & 12 \\
\hline Other valve surgery & \\
$\quad$ First assistant & 4 \\
\hline Total major cardiac procedures & First assistant \\
\hline Thoracotomy \pm lung biopsy & \\
\hline First assistant & 200 \\
\hline
\end{tabular}

the teaching facilities by 2 board members. The faculty must satisfy 7 standards prescribed by the RACS. The reaccreditation cycle is 3 to 5 years at the discretion of the Board depending on the recommendation of the previous accreditation. The trainee has the right and is encouraged by the board to report poor training/teaching facilities.

\section{REQUIRED OPERATIVE EXPERIENCE}

The RACS Board of Cardiothoracic Surgery has set criteria for minimum operative experience by the conclusion of training (Tables 2 and 3). Trainees are deemed eligible to sit for the fellowship examinations upon completion of $75 \%$ of the minimum operative experience. Depending on a trainee's progress, the duration of training may be prolonged, and this is at the discretion and approval of the RACS Board of Cardiothoracic Surgery. Because most units perform both cardiac and thoracic surgery, there can be variation in the duration of training in either domain, although a minimum of 12 months in a unit performing thoracic surgery is required. Although completion of the training program encompasses achieving operative case numbers, trainees who have obtained substantial operative experience before entering the formal training program may apply to the Board for recognition of prior learning, which if granted, can shorten the period of SET program training. The Board of Cardiothoracic Surgery favors competency- based training rather than time based, provided the trainee fulfills all the training requirements.

\section{RESPONSIBILITIES DELEGATED TO THE TRAINEE}

The degree of clinical and operative responsibility delegated to the trainee increases with seniority and depends largely on the trainee's level of competence with various tasks. In the first half of cardiac surgery training, trainees are generally tasked with gaining proficiency in peripheral conduit harvesting (saphenous vein, radial artery) before moving on to case components (ie, sternotomy, internal thoracic artery harvesting, cannulation for bypass, sternal closure).

Trainees are initially directly assisted by the supervising consultant, but may be permitted to perform components independently as they become more proficient and are deemed competent. Some trainees in their early years will perform entire cases (usually coronary artery bypass or aortic valve replacement), with the assistance of the consultant, depending on their ability. Likewise, in the early half of training in thoracic surgery, trainees will generally perform smaller procedures (thoracoscopy, bronchoscopy, decortication, wedge resection) with assistance.

In the latter half of training, additional levels of responsibility are given. In cardiac surgery, case components (sternotomy, conduit harvest, cannulation for bypass, closure) are generally performed independently with consultant assistance occurring during cardiopulmonary bypass and the period of aortic crossclamping. In the final year of training, trainees may perform entire cases independently (consultant surgeon present but unscrubbed). In thoracic surgery, trainees typically become proficient at major lung resection procedures (lobectomy, pneumonectomy), and again, in the final years of training will perform these independently with the consultant unscrubbed.

In the latter years of training, trainees also gain supervised operative experience in more complex procedures such as mitral valve surgery, surgery for atrial fibrillation, aortic surgery, transplantation, re-do sternotomy, complex thoracic surgery, and so forth. Regardless of seniority of the trainee and their operative and clinical contribution, the supervising consultant surgeon is ultimately responsible for the intraoperative and perioperative care of patients, and trainee autonomy is at the discretion of the consultant.

In addition to added operative responsibility, trainees are also encouraged to make a greater contribution to clinical decision-making, problem-solving, and management of complications. Senior trainees are also expected to lead departmental meetings, audits, journal clubs, research output, and the education of colleagues and junior staff.

\section{COURSES AND SIMULATION}

All RACS trainees must complete 3 RACS clinical skills courses. These are the Australian and New Zealand Surgical 
Skills Education and Training, Care of the Critically Ill Surgical Patient, and Early management of Severe Trauma courses. These courses are similar to trainee courses run in other countries. Care of the Critically Ill Surgical Patient and Early management of Severe Trauma provide a degree of clinical simulation and team exercises. In addition, cardiothoracic trainee workshops and skills laboratories are conducted at least twice per year. Supplementary industry-run wetlabs are also accessible to trainees. Crisis-management and team training modules are also available but are not mandatory components of training. Outside of formal courses and wetlabs, simulation still plays a limited role in day-to-day cardiothoracic training at present.

\section{ASSESSMENT}

As mentioned previously, all prospective trainees must pass the RACS GSSE examination before entering training in a RACS specialty. Within the first 2 years after commencement of training, the RACS Clinical Examination must also be completed. This examination tests candidates' clinical application of basic sciences as well as technical and nontechnical abilities. The exam is an Objective Structured Clinical Examination consisting of multiple stations on history-taking, physical examination, communication, and procedural skills.

In addition, a mid-training examination, the RACS Cardiothoracic Surgical Science and Principles examination, must be passed by the end of the fourth year of training. The written component tests candidates' knowledge of cardiovascular physiology, pathology, pharmacology, electrophysiology, and embryology, whereas an oral examination tests knowledge in anatomy relevant to Cardiothoracic Surgery.

The final RACS Fellowship Examination may be taken in the fifth or sixth year of training. This exit examination consists of written and multiple oral components, and focuses on the application of basic science knowledge, clinical knowledge, clinical skills, and management and decision-making in Cardiothoracic Surgery. Its purpose is to assess candidates' preparedness to commence practice as an independently competent cardiothoracic surgeon. The clinical component consists of 4 clinical cases, where candidates take a history and perform a physical examination of patients. In addition, three 40-minute viva-type stations present candidates with operative surgical and clinical scenarios. The viva components of the examination have evolved in recent years, with surgical anatomy and pathology oral examinations being removed and incorporated into the mid-training examination. The final clinical examination is thus more clinically focused with a greater emphasis on decisionmaking and higher-order thinking. The pass rate for the final fellowship examination ranges between $50 \%$ and $75 \%$.
In addition to the formal College examination, trainees are also assessed regularly in the workplace at the institutional level. A designated supervisor of training within a training unit assesses the trainee-in association with all surgeons within the department-every 6 months and provides feedback to the trainee and to the RACS Board of Cardiothoracic Surgery regarding progress, strengths, and areas for improvement. In addition to clinical and operative competence, trainees are also assessed on their professionalism, communication, and scholarship skills in line with the RACS' core values and 9 competencies. ${ }^{6}$

The RACS runs a course for consultant surgeons known as the Supervisors and Trainers for SET course to facilitate their role. This workshop helps surgeons understand their responsibilities, including legal issues surrounding assessment. It explores strategies that focus on the performance improvement of trainees, introducing the concepts of work-based training and work-based assessment tools.

Trainees and consultants must also complete regular Direct Observation of Procedural Skills (DOPS) assessments. These assessments allow consultants to assess trainees' level of competence in procedures or their components, such as conduit harvest, sternotomy, coronary artery bypass grafting, and aortic valve replacement to name a few. The DOPS assessments are standardized, with operative and nonoperative components of a procedure being considered. Trainees' competence is rated at one of 4 levels: (1) unable to perform; (2) able to partially perform under supervision; (3) able to perform with minimal supervision; (4) competent to perform unsupervised. The assessment process also permits consultants and trainees to provide feedback and to identify strengths, areas for improvement, or areas that are unsatisfactory. DOPS assessments provide guidance for trainees and supervisors to focus learning activities. The assessment documents are required to be submitted at regular intervals and are recorded centrally by the RACS Board of Cardiothoracic Surgery. Over the course of training, cardiothoracic trainees will typically complete 50 to 60 DOPS assessments.

In addition, all Cardiothoracic Surgery trainees must complete an investigative research project, publish at least 2 original articles in peer-reviewed journals, and submit a 5000- to 10,000 -word minor research thesis to the RACS Board of Cardiothoracic Surgery. This thesis is assessed by members of the Board or externally. Trainees who obtain a higher degree (ie, Masters, $\mathrm{PhD}$ ) before or during training are exempt from this thesis requirement.

Trainees who do not meet assessment requirements at any stage may be required to undertake a period of 3 to 6 months of probationary training, during which they are guided and closely supervised to develop their knowledge and skills in identified areas. The supervisor of training, in association with the department's staff, completes 
TABLE 4. Summary of requirements for completion of training in Cardiothoracic Surgery

Clinical

6 y of clinical rotations (undertaken at $\geq 3$ centers)

Satisfactory trainee evaluation at 6-mo intervals

Regular satisfactory completion of DOPS assessments by staff surgeons

Completion of minimum procedural logbook requirements

Completion of RACS skills courses and workshops

Academic

Publish 2 peer-reviewed manuscripts

Complete 10,000-word research thesis (or higher research degree)

Participation at annual ANZSCTS Scientific meeting

Examination

Generic surgical sciences examination (before entry into training)

Clinical examination (years 1-2 of training)

Cardiothoracic surgical science and Principles examination (years 2-4 of training)

Fellowship examination (years 5-6 of training)

$\overline{D O P S \text {, Direct observation of procedural skills; } R A C S \text {, Royal Australasian College of }}$ Surgeons; ANZSCTS, Australia and New Zealand Society of Cardiac and Thoracic Surgeons.

probationary assessment forms at regular intervals, and these are reviewed by the Board. If a trainee's clinical rotation has been assessed as unsatisfactory, the Board may require an extension to training.

\section{BOARD CERTIFICATION/ADMISSION TO FELLOWSHIP}

The requirements for completion of training in Cardiothoracic Surgery are presented in Table 4. Board certification, becoming a FRACS in Cardiothoracic Surgery, is the expected outcome after the completion of training and passing of the Fellowship Examination. This process is overseen by the RACS. On completion of the final examination and all other requirements of training, the trainee must obtain formal support from their current supervisor of training and the Chairperson of the RACS Board of Cardiothoracic Surgery. The Board Chairperson thereafter formally recommends to the RACS Censor-in-Chief that the trainee be awarded Fellowship. The process takes approximately 1 month to complete. Those who have been awarded FRACS in Cardiothoracic Surgery or any other specialty are deemed to be competent to practice independently and may assume consultant/faculty posts thereafter.

\section{CHALLENGES}

In Australia and New Zealand, a career in Cardiothoracic Surgery remains a relatively appealing option for junior doctors. Because of the limited number of training positions available per year and the relatively large pool of applicants, training positions are rarely unfilled.

The training program can take 9 to 10 years to complete from medical school graduation. Although this is ostensibly a long period of time, in Australia and New Zealand, junior consultants are expected to commence their specialist practice with a great deal of independence. The length of training is currently seen by the Cardiothoracic Surgery community to be appropriate to gain the knowledge and skills required for the level of autonomy and independence expected at junior consultant level.

As has been witnessed in cardiac surgery programs around the world, patients presenting for surgery are increasingly older, have a greater number of comorbidities, and represent higher operative risk than in the past. Indeed, the ideal training case of a young patient with 2-vessel disease and normal ventricular function is a rarity. In our center, trainee cardiac surgical cases are often older patients with diabetes, chronic kidney disease, and obesity, exhibiting diffusely diseased distal targets and requiring concomitant valve and coronary surgery. Cardiac surgery is also a heavily scrutinized specialty with respects to key performance indicators such as complications, duration of hospitalization, and costs. Therefore, the obligation to provide trainees with an adequate operative experience must be balanced with risk minimization, which is particularly difficult in higher-risk scenarios where the margins are narrow.

In Australia and New Zealand, training occurs almost exclusively in government-funded public hospitals that provide universal health care. These are distinct from private hospitals, which are funded by private companies and health insurers. Public hospitals have more limited resources in areas such as intensive care unit capacity/availability and after-hours staffing, yet are tasked with education and training. Cardiothoracic Surgery operating lists are often under pressure to finish within time constraints to minimize the costs of staff overtime pay; this places significant additional pressure on consultants and trainees.

Of the 30 training centers in Australia and New Zealand, the expectation and experiences of trainees can vary substantially. In some centers, trainees are supervised and assisted closely for all components of cases by consultants. However, in some larger centers, a greater degree of independence may be expected. Certain centers are more suited to junior trainees, and others are suited to those more senior. Changes in consultant personnel can also alter the complexion of a unit with respect to training. Although the RACS Board of Cardiothoracic Surgery generally accounts for these factors, a training post may not always be well suited to a trainee's experience level. The presence of local and international trained postgraduate fellows, looking to gain further operative experience, can also affect the experience of the trainee, especially in units with smaller caseloads.

In the first year of training, Cardiothoracic Surgery trainees are required to spend a period of time within the hospital's Cardiology department. Trainees' experiences 
have been variable, although some have reported positive experience such as performing diagnostic catheterization and basic interventional and structural heart procedures under the supervision of a cardiologist. Incorporation of transcatheter and wire-skills into the Australian and New Zealand Cardiothoracic Surgery curriculum is currently being pursued.

\section{CONCLUSIONS}

Cardiothoracic Surgery training in Australia and New Zealand is a rigorous, high-quality process involving regular in-training assessments and multiple examinations spread over a period of at least 6 years. Trainees typically spend time at 3 to 4 different centers, gaining proficiency in both adult cardiac and general thoracic surgery. Training and subsequent admission to Fellowship are overseen centrally by the RACS, and such grants individuals the privilege to practice independently as a consultant cardiothoracic surgeon. The training curriculum is expected to continually evolve to optimally equip trainees for contemporary practice.

\section{Conflict of Interest Statement}

Authors have nothing to disclose with regard to commercial support.

The authors thank Sue Moshis, Executive Officer of the RACS Board of Cardiothoracic Surgery, for assistance with preparation of the manuscript.

\section{References}

1. Clarebrough J. History of early cardiac surgery in Australia. Heart Lung Circ 2003;12(Suppl 1):S15-20.

2. Stats NZ. Population. Available at: https://www.stats.govt.nz. Accessed January $10,2018$.

3. Australian Bureau of Statistics. Australian demographic statistics. Available at: http://www.abs.gov.au/. Accessed January 10, 2018.

4. Australia and New Zealand Society of Cardiac and Thoracic Surgeons. ANZSCTS national database. Available at: https://anzscts.org/database/. Accessed January $10,2018$.

5. Nag N, Turbic A, Shardey G, Baker R, Newcomb A, Reid C, et al. The Australian and New Zealand Society of Cardiac and Thoracic Surgeons Cardiac Surgery Database Program: National Annual Report. Available at: https://anzscts.org/ database/about/\#reports. Accessed January 10, 2018.

6. Royal Australasian College of Surgeons. Competencies. Available at: https:// www.surgeons.org/becoming-a-surgeon/surgical-education-training/competencies/. Accessed January 10, 2018. 